\title{
Accelerating proton spin diffusion in perdeuterated proteins at 100 $\mathrm{kHz}$ MAS
}

\section{Journal Article}

Author(s):

Wittmann, Johannes J.; Agarwal, Vipin; Hellwagner, Johannes; Lends, Alons; Cadalbert, Riccardo; Meier, Beat H.; Ernst, Matthias (iD)

Publication date:

2016-12

\section{Permanent link:}

https://doi.org/10.3929/ethz-b-000122377

Rights / license:

In Copyright - Non-Commercial Use Permitted

Originally published in:

Journal of Biomolecular NMR 66(4), https://doi.org/10.1007/s10858-016-0071-8 


\section{Accelerating proton spin diffusion in perdeuterated proteins at $100 \mathrm{kHz}$}

MAS

Johannes J. Wittmann ${ }^{\mathrm{a}}$, Vipin Agarwal ${ }^{\mathrm{a}, \mathrm{b}}$, Johannes Hellwagner ${ }^{\mathrm{a}}$, Alons Lends ${ }^{\mathrm{a}}$, Riccardo Cadalbert ${ }^{\mathrm{a}}$, Beat H. Meier ${ }^{\mathrm{a}^{*}}$, Matthias Ernst $\mathrm{t}^{\mathrm{a}^{*}}$

aphysical Chemistry, ETH Zurich, Vladimir-Prelog-Weg 2, 8093 Zurich, Switzerland

${ }^{b}$ TIFR Center for Interdisciplinary Science, 21 Brundavan Colony, Narsingi, Hyderabad 500075, India

\section{Corresponding Authors:}

Beat H. Meier, Matthias Ernst

Physical Chemistry, ETH Zürich, Vladimir-Prelog-Weg 2, 8093 Zürich, Switzerland

E-mail: beme@ethz.ch,maer@ethz.ch

Phone: +41 4463244 01, +41446324366 


\begin{abstract}
Fast magic-angle spinning ( $>60 \mathrm{kHz}$ ) has many advantages but makes spin-diffusion-type proton-proton long-range polarization transfer inefficient and highly dependent on chemical-shift offset. Using $100 \%$ $\mathrm{HN}-\left[{ }^{2} \mathrm{H},{ }^{13} \mathrm{C},{ }^{15} \mathrm{~N}\right]$-ubiquitin as a model substance, we quantify the influence of the chemical-shift difference on the spin diffusion between proton spins and compare two experiments which lead to an improved chemical-shift compensation of the transfer: rotating-frame spin diffusion and a new experiment, reverse amplitude-modulated MIRROR. Both approaches enable broadband spin diffusion, but the application of the first variant is limited due to fast spin relaxation in the rotating frame. The reverse MIRROR experiment, in contrast, is a promising candidate for the determination of structurally relevant distance restraints. The applied tailored rf-irradiation schemes allow full control over the range of recoupled chemical shifts and efficiently drive spin diffusion. Here, the relevant relaxation time is the larger longitudinal relaxation time, which leads to a higher signal-to-noise ratio in the spectra.
\end{abstract}

\title{
Keywords
}

fast MAS, protein structure determination, spin diffusion 


\section{Introduction}

The coherent averaging of anisotropic interactions by magic-angle spinning (MAS) (Andrew et al. 1958; Lowe 1959) is a prerequisite for obtaining high-resolution NMR spectra of polycrystalline samples. In general, higher MAS frequencies lead to a better averaging of anisotropic interactions and, therefore, to higher spectral resolution especially in proton spectra. For inhomogeneous interactions, i.e., interactions where the Hamiltonian commutes with itself at all times, MAS frequencies on the order of the interaction are usually sufficient to obtain well-resolved spectra (Maricq and Waugh 1979). Even though recent technological advances in probe development allow spinning frequencies above $110 \mathrm{kHz}$ (Nishiyama et al. 2012; Parthasarathy et al. 2013; Agarwal et al. 2014; Deschamps 2014), the homogeneous broadening caused by the strong dipolar-coupled proton-proton network in bio-macromolecules is still only partially averaged out (Zhou et al. 2007; Bertini et al. 2011; Lewandowski et al. 2011). Consequently, a significant residual line-broadening is observed due to non-vanishing higher-order terms in the expansion of the average Hamiltonian (Böckmann et al. 2015). A further increase in the resolution of proton spectra can be obtained by isotope dilution of the protons, e.g. per-deuteration of the protein (Chevelkov et al. 2006; Akbey et al. 2010; Reif 2012). Besides an improved spectral resolution, fast MAS has the additional benefit that low-power experiments (Ernst et al. 2004; Lange et al. 2009; Demers et al. 2011; Teymoori et al. 2012; Agarwal et al. 2013) can be implemented, i.e., experiments where the nutation frequency of the typical rf fields is lower than the MAS frequency. The reduced rf power causes less sample heating, enabling faster repetition rates and, consequently, an increased sensitivity per unit time.

Despite the multiple advantages of fast MAS, a major problem in its application to biomolecular NMR is the measurement of structural restraints. At slow to intermediate spinning frequencies, a number of second-order recoupling sequences have been developed over the years (Szeverenyi et al. 1982; Takegoshi et al. 2001; Lange et al. 2002; Takegoshi et al. 2003; Grommek et al. 2006; De Paëpe et al. 2008; Duma et al. 2008; Scholz et al. 2008; Weingarth et al. 2009; Ladizhansky 2009; Weingarth et al. 2010; Hu et al. 2011; Hou et al. 2013; Wittmann et al. 2014). As these experiments rely on the higher-order terms in the 
average Hamiltonian, they are less susceptible to dipolar-truncation effects (Hohwy et al. 2002; Bayro et al. 2009; Ladizhansky 2009) and can be used to acquire long-range distance restraints between X-nuclei, like ${ }^{13} \mathrm{C}$ or ${ }^{15} \mathrm{~N}$. However, the efficiency of these recoupling sequences scales inversely with the MAS frequency. As a consequence, the polarization transfer in second-order recoupling sequences between Xnuclei slows down significantly at fast MAS. In principle, longer mixing times can compensate for a slower polarization transfer, but an overall loss of signal due to relaxation effects will always be observed.

For proton-detected spectra that become attractive above $60 \mathrm{kHz}$ MAS in (partially) deuterated proteins, it seems natural to also acquire distance restraints between protons to achieve faster polarization transfer and to minimize the number of polarization-transfer steps (Penzel et al. 2015). It was shown that first-order recoupling sequences, such as RFDR (Bennett et al. 1998; Ishii 2001; Zhou et al. 2007; Linser et al. 2011) or DREAM (Verel et al. 2001; Huber et al. 2011), provide a reliable set of distance restraints in perdeuterated proteins with $100 \%$ exchangeable protons or in methyl-protonated systems at MAS frequencies up to $60-100 \mathrm{kHz}$. However, relatively high rf-field amplitudes are often required, which could lead to a degradation of the sample by induced heating. In addition, both first-order recoupling sequences are susceptible to dipolar truncation, which will increase with higher protonation levels. Therefore, secondorder recoupling sequences are still desirable when determining proton-proton distances. The simplest second-order sequence is proton spin diffusion as used in the $\mathrm{CHHC}$ and $\mathrm{NHHC}$ experiment for ${ }^{13} \mathrm{C}$ detected spectra (Lange et al. 2002). However, at MAS frequencies above $60 \mathrm{kHz}$, even proton spin diffusion shows a strong dependence on the chemical-shift difference due to the significantly reduced overlap of the single-quantum lines in the spectrum (vide infra). Rotating-frame spin diffusion (Robyr et al. 1989) is one possible solution to this problem (Agarwal et al. 2014), but it has the disadvantage that the relaxation times in the rotating frame are typically much shorter than in the laboratory frame.

In the following, we propose a novel approach for chemical-shift compensated proton spin diffusion at fast MAS frequencies above $60 \mathrm{kHz}$ that yields high transfer efficiencies in per-deuterated proteins (with $100 \%$ protonation at exchangeable sites). The new scheme is based on the principles of the recently 
proposed AM-MIRROR recoupling (Wittmann et al. 2014) with the rf irradiation applied to the heteronuclear spins (here: ${ }^{15} \mathrm{~N}$ ). A quantitative comparison of the efficiency of AM-MIRROR with labframe and rotating-frame proton-proton spin-diffusion mixing is presented.

\section{Theoretical Considerations for Spin Diffusion under MAS}

The classical approach to describe spin diffusion under MAS is based on perturbation theory (Kubo and McDowell 1988; Veshtort and Griffin 2011) where the rate constants $k_{i j}$ for spin diffusion between two spins $i$ and $j$ are expressed as

$$
k_{i j}=\sum_{n=-2}^{2} \omega_{i j}^{(n)} \omega_{i j}^{(-n)} J\left(n \omega_{\mathrm{r}}+\Delta \Omega_{\mathrm{iso}}\right)
$$

There are two parameters, which influence the magnitude of the spin-diffusion rate constants $k_{i j}$ : (i) the dipolar coupling $\omega_{i j}$ and (ii) the chemical-shift difference $\Delta \Omega_{\text {iso }}$ between the two coupled spins. While the

dependence on the coupling constant scales with the square of the Fourier components $\omega_{i j}^{(n)}$ of the dipolar coupling, the chemical-shift difference influences Eq. (1) indirectly via the frequency $\omega$ at which the intensity of the normalized zero-quantum line $J(\omega)$ is sampled. Assuming that the broadening of the two one-quantum resonances is uncorrelated, the zero-quantum line $J(\omega)$ is a measure for the spectral overlap of the resonances and can be calculated as the convolution of the two single-quantum lines (Kubo and McDowell 1988). With increasing MAS frequency, the side-band intensity of the zero-quantum line, which is the relevant contribution, scales as $1 / \omega_{\mathrm{r}}$ and, furthermore, the zero-quantum line becomes narrower. Therefore, spin-diffusion slows down for larger shift differences and becomes strongly dependent on the chemical-shift difference with increasing MAS frequency. A schematic representation of a zero-quantum line under fast magic-angle spinning conditions is shown in Figure 1A. 

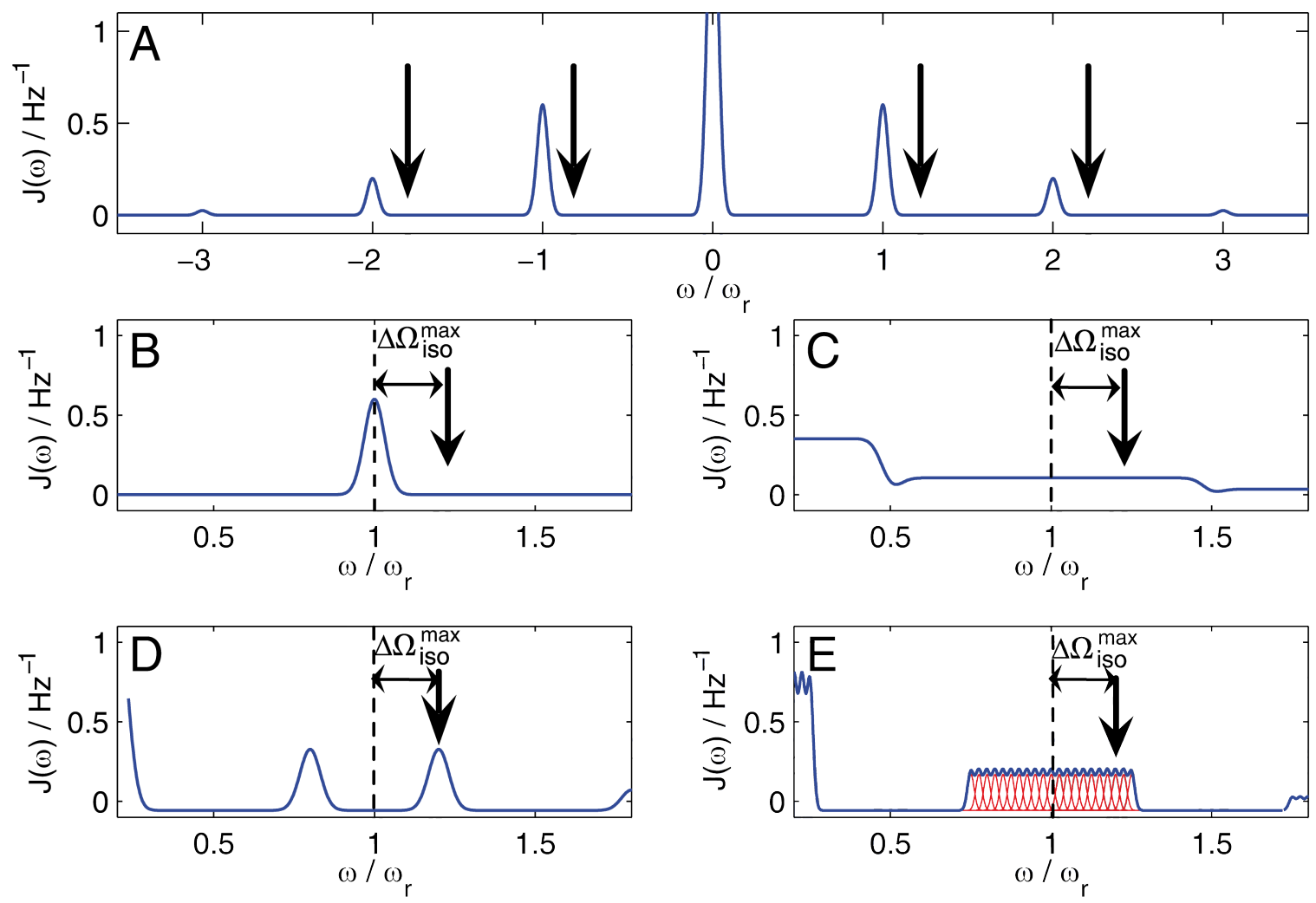

Figure 1: Schematic representation of a zero-quantum line for laboratory-frame spin diffusion under fast MAS. A) The zero-quantum line consists of a central band and several MAS side bands. B) Enlarged view of the first MAS side band for conventional laboratory-frame spin diffusion. In the fast-spinning limit the intensity quickly drops to zero for $\boldsymbol{\Delta} \boldsymbol{\Omega}_{\text {iso }}>\mathbf{0}$ explaining the strong chemical-shift dependence. C) The MAS side bands can be broadened by dipolar recoupling (e.g. in the DARR experiment) to promote broad-band spin diffusion. However, the width of this broadening is determined by the largest recoupled interaction and, thus, it cannot be adjusted to optimally cover the relevant chemical-shift ranges. In the case shown, the range of recoupled chemical shifts is larger than necessary, leading to inefficient transfer. In the MIRROR experiment additional rf side bands are generated at multiples of the basic frequency leading to a zero-quantum line shape that promotes band-selective (D, CW-MIRROR) or chemical-shift independent spin diffusion (E, AM-MIRROR). In the given example (E), several rf sidebands (red curves) overlap to form a quasi-continuous distribution of the available zero-quantum intensity and spin diffusion is recoupled for $\boldsymbol{\Delta} \boldsymbol{\Omega}_{\text {iso }} \lesssim \Delta \boldsymbol{\Omega}_{\text {iso }}^{\max }$ with uniform rates. The black arrows indicate the sampling contributions for the calculation of $k_{i j}$ according to Eq. (1) at a chemical shift of $\Delta \Omega_{\text {iso }}^{\max }=0.2 \omega_{\mathrm{r}}$.

An equivalent description can be obtained based on a Floquet analysis, which offers a quantitative way to access the terms driving the polarization transfer under MAS. The relevant contributions for proton-proton spin diffusion in the laboratory-frame are three-spin terms contained in the second-order effective Hamiltonian (Scholz et al. 2010). They are of the general form 


$$
\omega_{\text {eff }}\left(\omega_{i j}^{(n)}, \omega_{i p}^{(n)}, \omega_{j p}^{(n)}\right) S_{p z}\left(I_{i}^{+} I_{j}^{-}-I_{i}^{-} I_{j}^{+}\right)
$$

and

$$
\omega_{\mathrm{eff}}\left(\omega_{i p}^{(n)}, \omega_{j p}^{(n)}, \omega_{i j}^{(n)}\right) I_{p z}\left(I_{i}^{+} I_{j}^{-}-I_{i}^{-} I_{j}^{+}\right) .
$$

The spin operators are defined according to Scholz et al. 2010 and $i, j$, and $p$ characterize three different but dipolar-coupled spins with the Fourier coefficients of the dipolar couplings $\omega_{i p}^{(n)}, \omega_{i j}^{(n)}$, and $\omega_{j p}^{(n)}$. The magnitude $\omega_{\text {eff }}$ of these terms is given by cross-terms between a heteronuclear ${ }^{1} \mathrm{H}-\mathrm{X}$ (with $\mathrm{X}$ being for example ${ }^{13} \mathrm{C}$ or ${ }^{15} \mathrm{~N}$ ) and a homonuclear ${ }^{1} \mathrm{H}-{ }^{1} \mathrm{H}$ dipolar coupling or by products of three homonuclear proton-proton couplings, respectively (Scholz et al. 2010). These terms scale inversely with the MAS frequency.

The magnitude of the truncation terms induced by the chemical shift is independent of the MAS frequency, as these fictitious fields, which interfere with the recoupling, in the effective Hamiltonian originate from non-resonant contributions in first-order approximation. There are two different approaches to overcome this chemical-shift dependence of proton spin diffusion. On the one hand, the chemical-shift differences can be quenched by irradiation of a rf field on the proton channel (Robyr et al. 1989) which is known as rotating-frame spin diffusion. The spin lock eliminates the perturbing terms proportional to the isotropic chemical shift from the non-resonant term in the Hamiltonian. Consequently, spin diffusion in the rotating frame relies on cross terms by homonuclear dipolar interactions only (Eq. (3)), as the spin part of the heteronuclear dipolar interaction acquires an additional time dependence due to the rf field irradiation. This approach has extensively been used for structure elucidation in static solids (Robyr et al. 1991; Meier 1994; Kümmerlen et al. 1996; Senker et al. 2004). For rotating samples, i.e., under MAS, its application is limited especially at slow MAS frequencies, as the applied rf field must not be close to any of the first-order resonance conditions (Ernst et al. 2005).

On the other hand, the shape of the zero-quantum line can be altered by irradiating coupled heteronuclear spins with a radio-frequency field as in the DARR (Takegoshi et al. 2001; Takegoshi et al. 2003) or 
MIRROR (Scholz et al. 2008) experiment. In the perturbation-theory approach, the DARR experiment, where the proton rf-field amplitude is matched to the spinning frequency, causes a broadening of the zeroquantum line (Figure 1C) due to dipolar recoupling (Takegoshi et al. 2001; Takegoshi et al. 2003). Alternatively, the irradiation of MIRROR-type experiments (involving cw, phase- or amplitude-modulated rf fields on the proton channel) causes the generation of additional rf side bands in the zero-quantum spectrum (Duma et al. 2008; Scholz et al. 2008; Weingarth et al. 2009; Weingarth et al. 2010; Hou et al. 2011; Hou et al. 2013; Wittmann et al. 2014). These side bands appear at multiples of the basic frequency $\omega_{\mathrm{m}}$ of the recoupling sequence and are caused by the modulation of the heteronuclear dipolar coupling (Scholz et al. 2008). Depending on the applied rf sequence, band-selective or broad-band spin diffusion can be achieved (Figure 1D and E). In all of the cases, the polarization exchange occurs when the secondorder resonance condition

$$
n \omega_{\mathrm{r}}+k \omega_{\mathrm{m}}+\Delta \Omega_{\mathrm{iso}}=0
$$

is fulfilled and terms proportional to

$$
\overline{\mathcal{H}}_{\mathrm{eff}}^{(2)} \propto \omega_{\mathrm{eff}}\left(\omega_{i j}^{(n)}, \omega_{i k}^{(n)}, \omega_{j k}^{(n)}\right) a_{ \pm k} S_{p}^{ \pm}\left(I_{i}^{+} I_{j}^{-}-I_{i}^{-} I_{j}^{+}\right)
$$

drive the polarization transfer (Wittmann et al. 2014). The magnitude of the effective Hamiltonian is dependent not only on the cross terms between dipolar interactions but also on the Fourier coefficients $a_{ \pm k}$. These can be calculated from the applied rf field and, thus, tailored irradiation schemes can be designed which most efficiently distribute the available zero-quantum intensity over previously selected chemical-shift ranges (Wittmann et al. 2014).

All of these techniques have previously been applied to ${ }^{13} \mathrm{C}$ or ${ }^{15} \mathrm{~N}$ spin diffusion under MAS (Duma et al. 2008; Scholz et al. 2008; Weingarth et al. 2009; Weingarth et al. 2010; Hou et al. 2011; Hou et al. 2013; Wittmann et al. 2014). We propose to use such techniques in a reverse manner to drive proton spin diffusion in the limit of fast MAS, for example, by irradiation on the ${ }^{15} \mathrm{~N}$ or ${ }^{13} \mathrm{C}$ channel. At $100 \mathrm{kHz}$ MAS, the application of a reverse DARR or phase-alternating recoupling experiment (like PARIS 
(Weingarth et al. 2010), SHANGHAI (Hu et al. 2011) or CORD (Hou et al. 2013)) is limited, as unfavorably high rf-field amplitudes are required to match the recoupling conditions $\left(\omega_{1}=\omega_{\mathrm{r}}\right.$ or $\omega_{1}=$ $\left.0.5 \omega_{\mathrm{r}}\right)$. Additionally, the achieved broadening of the zero-quantum lines is often much larger than required by the narrow chemical-shift range $(10 \mathrm{ppm})$ of the protons (Figure 1C). In contrast to these experiments, the AM-MIRROR experiment (Wittmann et al. 2014) allows a tailored broadening of the zero-quantum line (Figure 1E) as position and intensity of the rf sidebands in the zero-quantum spectrum can be predicted from the rf irradiation scheme. This relationship is used to design amplitude-modulated rf fields, which distribute all the available zero-quantum line intensity uniformly in the spectral ranges of interest leading to optimized spin diffusion. The maximum rf-field amplitude is only determined by the chemical-shift differences to be recoupled and does not depend on the MAS frequency. The AMMIRROR experiment can, therefore, be implemented as a low-power experiment. For example, in the case of spin diffusion among amide protons in proteins the maximum rf-field amplitude can be kept below $3 \mathrm{kHz}$ allowing long mixing times.

\section{Experimental}

All experiments were carried out on a Bruker Avance III spectrometer at a proton Larmor frequency of 850.15 MHz (20 T magnetic field). A $0.7 \mathrm{~mm}$ triple-resonance MAS probe (Bruker Biospin) was used for spinning the sample at $100 \mathrm{kHz}$. The $\mathrm{HNH} 3 \mathrm{D}$ pulse sequence is shown in Figure 2 with experimental details. For quantification of the transfer efficiencies, an NNH 3D experiment with higher spectral resolution was used as shown in Figure S1 of the Supporting Information. The mixing time was set to $20 \mathrm{~ms}$ for reverse MIRROR mixing and lab-frame spin diffusion, and to $10 \mathrm{~ms}$ for rotating-frame spin diffusion. In all the cases the mixing time was optimized to give maximum polarization transfer for amide protons one-residue apart. In order to avoid resonant effects with the MAS frequency, the amplitude of the spin-lock field for spin-diffusion in the rotating frame was experimentally optimized to $13 \mathrm{kHz}$. The AMMIRROR irradiation scheme $\left(v_{\mathrm{m}}=200 \mathrm{~Hz}, \tau_{\mathrm{m}}=5 \mathrm{~ms}\right)$ was optimized numerically as described before (Wittmann et al. 2014). Target function for the optimizations was a uniform distribution of rate-constants 
over a chemical-shift difference of $\pm 2 \mathrm{kHz}$. Amplitudes and phases of the scheme are provided as Bruker shape file in the supporting information (Table S2) for a $20 \mathrm{~T}$ static magnetic field. Optimized shapes for other magnetic fields or different chemical-shift ranges can be obtained by following the optimization procedures outlined in (Wittmann et al. 2014). $100 \%-\mathrm{HN}-\left[{ }^{2} \mathrm{H},{ }^{13} \mathrm{C},{ }^{15} \mathrm{~N}\right]$-ubiquitin was prepared by overexpression of uniformly ${ }^{2} \mathrm{H},{ }^{13} \mathrm{C},{ }^{15} \mathrm{~N}$-labeled ubiquitin in E. coli. After crystallization in protonated MPD with $\mathrm{H}_{2} \mathrm{O}$ to re-protonate the exchangeable sites (Igumenova et al. 2004) the sample was filled into $0.7 \mathrm{~mm}$ Bruker rotors by ultracentrifugation using a home-built device (Böckmann et al. 2009).

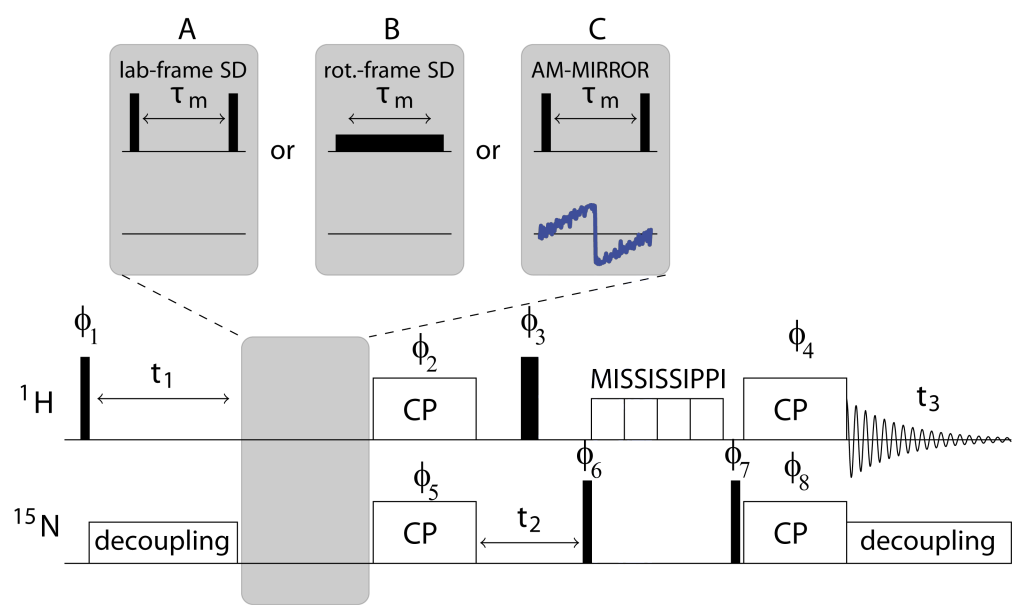

Figure 2: 3D HNH-correlation experiment used to compare the efficiency of the three proton spindiffusion experiments A) laboratory-frame spin diffusion, B) rotating-frame spin diffusion and C) AMMIRROR. The correlation of two proton dimensions in this experiment allows a direct and simple visual comparison of the dependence of the transfer on the chemical-shift difference. The spectra were recorded using 128 complex points in the $t_{1}\left({ }^{1} \mathrm{H}\right), 100$ complex points in the $t_{2}\left({ }^{15} \mathrm{~N}\right)$ and 2048 complex points in the $t_{3}\left({ }^{1} \mathrm{H}\right)$ dimension. States-TPPI was used for phase-sensitive detection in the indirect dimensions. The spectral widths of the individual dimensions were 6,37 and $47 \mathrm{ppm}$, respectively. ${ }^{15} \mathrm{~N}$ decoupling was achieved using a WALTZ-64 scheme at an rf field of $5 \mathrm{kHz}$. The CP contact time was set to $600 \mu \mathrm{s}$ to transfer only between directly bonded ${ }^{15} \mathrm{~N}$ and ${ }^{1} \mathrm{H}$ nuclei. Phase cycle: $\boldsymbol{\phi}_{1}: 0,0,2,2 ; \boldsymbol{\phi}_{2}: 1,1,1,1 ; \boldsymbol{\phi}_{3}: 0$; $\boldsymbol{\phi}_{\mathbf{4}}: \mathbf{0} ; \boldsymbol{\phi}_{\mathbf{5}}: 0,0,2,2 ; \boldsymbol{\phi}_{\mathbf{6}}: 3,3,1,1 ; \boldsymbol{\phi}_{7}: 1,3,3,1 ; \boldsymbol{\phi}_{\mathbf{8}}: 0,0,2,2 ; \boldsymbol{\phi}_{\text {rec }}: 0,2,2,0$;

\section{$4 \quad$ Results and Discussion}

Figure 3 shows representative planes and slices through a three-dimensional HNH-correlation spectrum of $100 \%-\mathrm{HN}-\left[{ }^{2} \mathrm{H},{ }^{13} \mathrm{C},{ }^{15} \mathrm{~N}\right]$-ubiquitin using $20 \mathrm{~ms}$ proton spin diffusion (Figure 3A), rotating-frame spin diffusion with a spin-lock field of $13 \mathrm{kHz}$ (Figure 3B) and AM-MIRROR spin diffusion with an N-H optimized irradiation scheme (Figure 3C) during the mixing time. For the pulse sequences see Figure 2. In 
case of conventional lab-frame spin diffusion (Figure 3A), all the observed cross peaks can be assigned to transfer to the amide protons of directly neighboring residues. However, a strong influence of the chemical-shift difference on the observed cross-peak intensity is visible. In the case of rotating-frame spin diffusion (Figure 3B) and AM-MIRROR spin diffusion (Figure 3C) this effect is much less pronounced. This can be seen more clearly in Figure 3D-J, where 1D traces through the 3D spectrum are shown. It is obvious that the cross-peak intensities (transferred polarization) are higher in both the rotating-frame and in the AM-MIRROR spectrum, but the overall intensity of the rotating-frame spin diffusion spectrum is significantly lower than the one of the two lab-frame experiments due to faster relaxation (see also Figure 4).
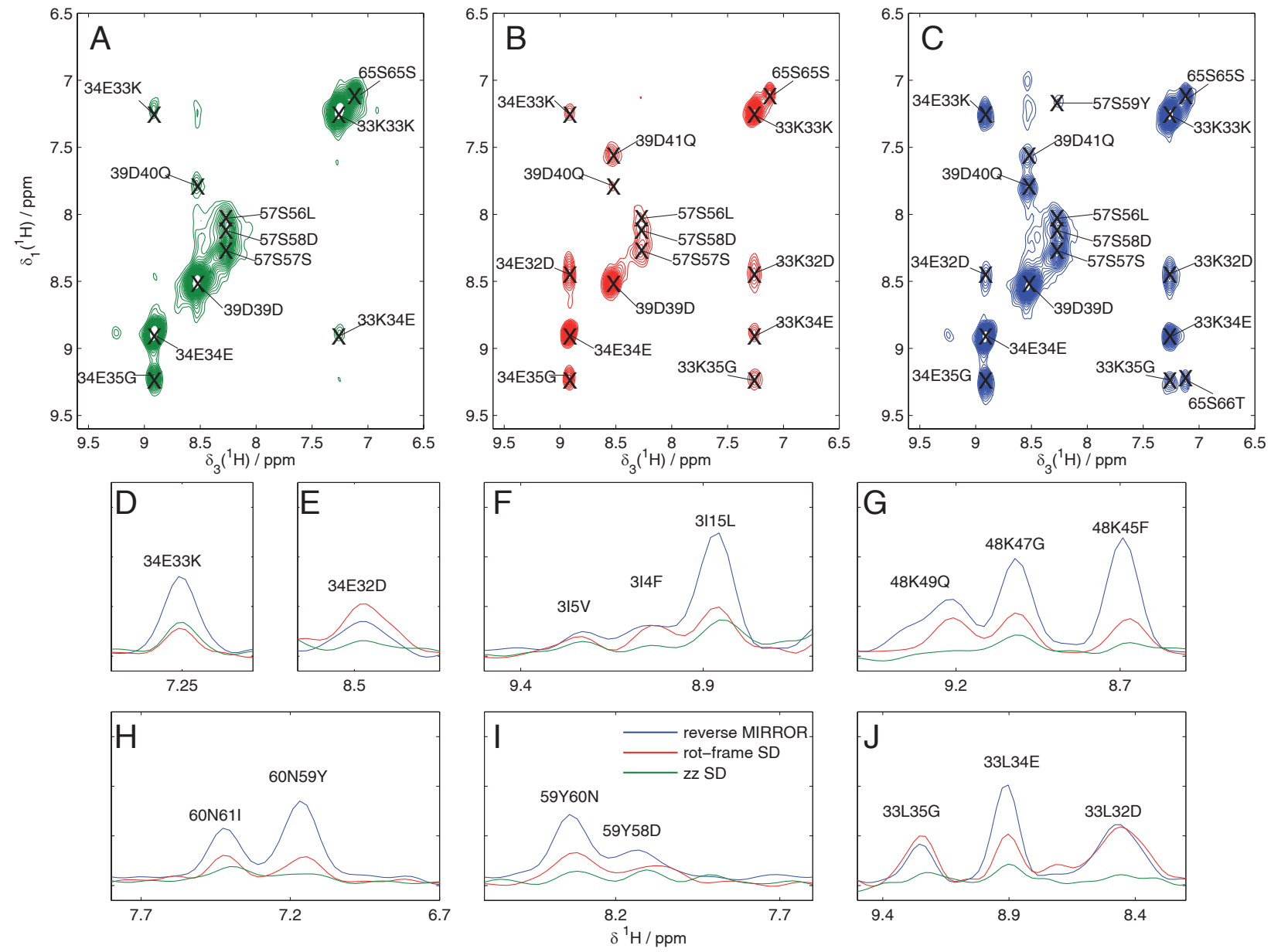

Figure 3: A-C) Representative planes through the 3D HNH-correlation spectra at $\boldsymbol{\delta}_{\mathbf{2}}\left({ }^{15} \mathbf{N}\right)=$ $112.8 \mathrm{ppm}$ illustrating the transfer efficiencies of the three mixing schemes. The conventional spin diffusion (A, green) shows the least efficiency and lacks stabilization against differences in chemical shift. 
Both, rotating-frame $(B$, red) and reverse MIRROR ( $C$, blue) spin diffusion are more broadband. However, reverse MIRROR yields significantly more intense cross peaks. Further planes are shown in Figures S2 of the Supporting Information. D-J) Selected traces through the 3D correlation spectra with several observed correlations.

In order to illustrate the dependence of the transfer efficiency on the chemical-shift difference in a systematic way, the normalized cross-peak intensity of the transfers to the neighboring residues is shown as a function of the proton chemical-shift difference for the three different mixing schemes in Figure 5. For a better quantification of the transfer the higher spectral resolution of an NNH-experiment (for the pulse sequence see Figure S1 of the Supporting Information) was exploited here. As the average amideamide proton distances differ significantly in different secondary-structure elements, we further distinguish between transfer in $\alpha$-helical (Figure 5A-C) and $\beta$-sheet domains (Figure 5D-F), respectively. In the case of laboratory-frame spin diffusion (Figure 5A and D), the qualitative dependence of transfer efficiencies as a function of chemical-shift differences is similar for both structural elements.

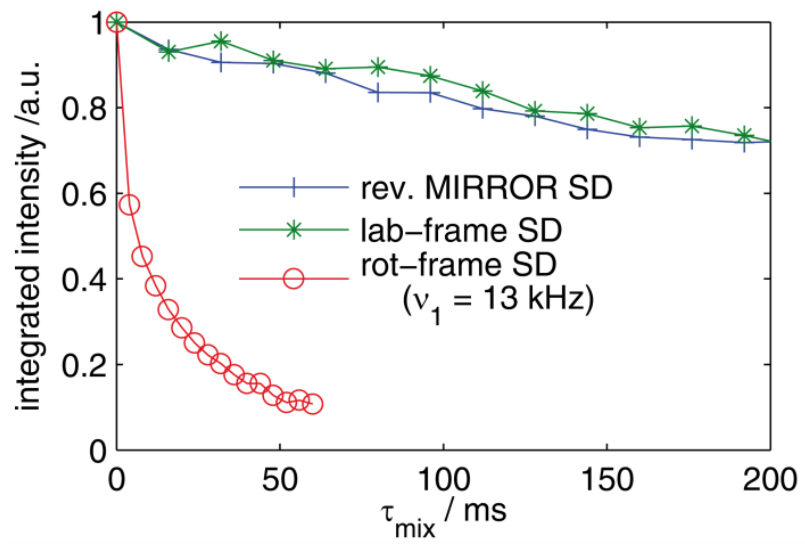

Figure 4: Comparison of the decay of the total magnetization (first point of fid) as a function of the mixing time for the three different polarization transfer schemes. During the spin-lock experiment, the magnetization decays much faster leading to a severe loss of total signal intensity at relevant mixing times.

For laboratory-frame spin diffusion, efficient transfer is only observed between resonances separated by small chemical-shift differences and a substantial truncation of the polarization transfer is observed for $\Delta v_{\text {iso }}>500 \mathrm{~Hz}$. Such a strong dependence on the chemical-shift difference is already found at spinning 
frequencies starting around $60 \mathrm{kHz}$. This is a consequence of the fact that fast MAS narrows down the zero-quantum lines significantly in the less strongly coupled proton network of the deuterated protein. In full analogy to proton-driven ${ }^{13} \mathrm{C}$ spin diffusion experiments at fast MAS, efficient transfer is only observed if the chemical-shift difference is smaller than the width of the zero-quantum line. Under our experimental conditions, the broadening by the residual proton-proton interactions is only strong enough to assist the polarization transfer as long as $\Delta v_{\text {iso }} \lesssim 200 \mathrm{~Hz}$. Note, that under the assumption that the mixing time of $20 \mathrm{~ms}$ is still in the initial-rate approximation, the obtained cross-peak intensities of Figure 5 directly map out the shape of the zero-quantum line $J(\omega)$ of Eq. (1).
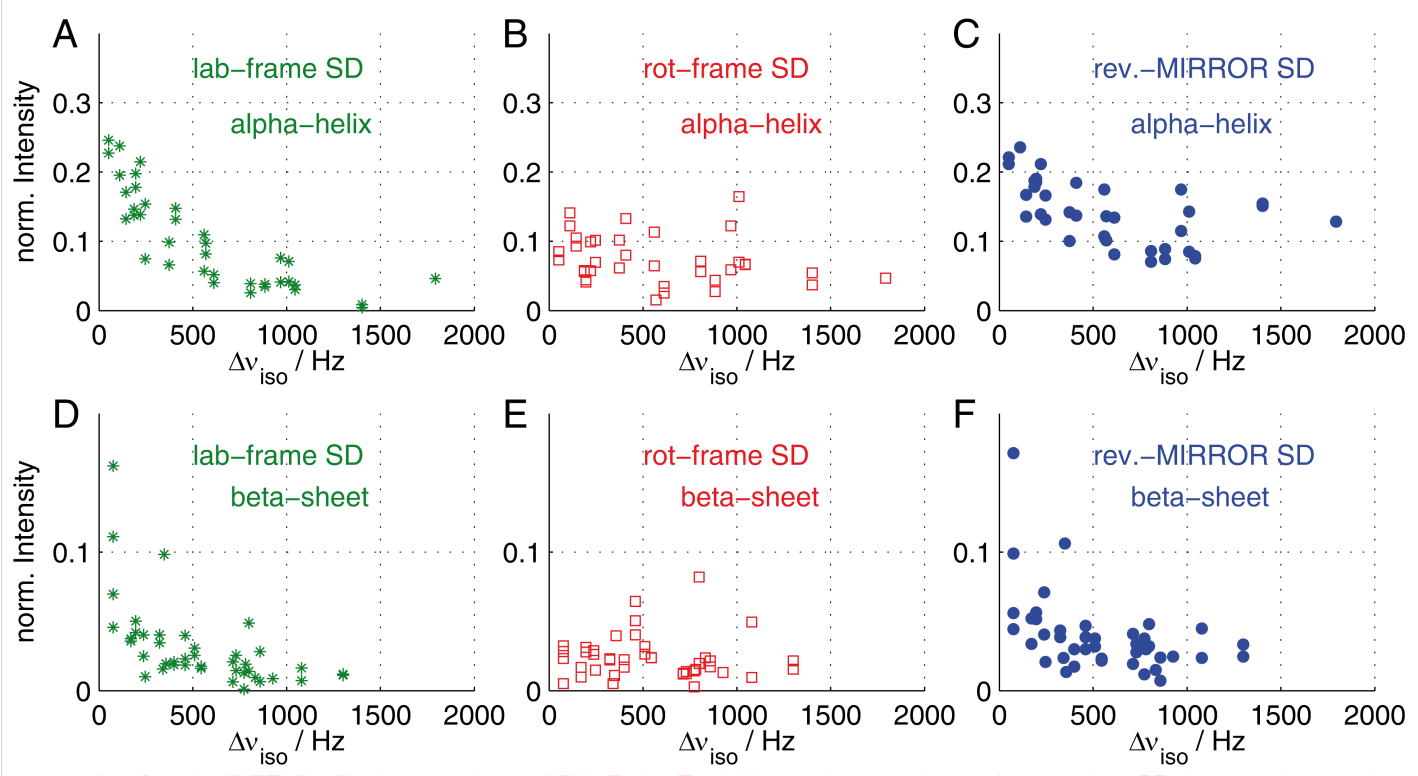

Figure 5: Intensities of cross-peaks in $100 \%-\mathrm{HN}-\left[{ }^{2} \mathrm{H},{ }^{13} \mathrm{C},{ }^{15} \mathrm{~N}\right]$-ubiquitin corresponding to transfer between the amide protons of neighboring residues in the $\alpha$-helical domains (top row) and $\beta$-sheets (bottom row) using three different mixing schemes. A and D) $20 \mathrm{~ms}$ laboratory-frame spin diffusion, B and E) $10 \mathrm{~ms}$ rotating-frame spin-diffusion and $\mathrm{C}$ and F) $20 \mathrm{~ms}$ reverse MIRROR. The intensities are taken from 3D NNH-correlation experiments and normalized to the signal intensity of the respective diagonal peak without mixing. The mixing times were optimized to give maximum cross-peak intensity for both experiments. A comparison of the intensity of all the observed cross peaks is shown in Figure S3 of the Supporting Information, for a comparison to RFDR and DREAM see Figure S4.

For rotating-frame spin diffusion one has to avoid resonance conditions, which could lead to an even faster decay of the spin-locked magnetization. Using an experimentally optimized rf-field amplitude of $13 \mathrm{kHz}$, transfer to residues separated by a significant chemical-shift difference is re-established as can be 
seen in Figure 5B and E. However, the overall intensity of the cross peaks - as well as the diagonal peaks - is relatively small. Nevertheless, this experiment leads to a gain in transfer efficiency to resonances separated by larger chemical-shift differences. The reason for the loss of approximately $40 \%$ of the total magnetization during the $10 \mathrm{~ms}$ spin lock are the relatively short $T_{1 \rho}$ relaxation times, which are in the same order of magnitude as the required spin-diffusion mixing times (see Figure 4). This poor sensitivity is the major drawback for using rotating-frame spin diffusion as a polarization-transfer mechanism in proteins. Despite the fast transfer, the rapid relaxation limits the experimentally feasible mixing times to below $\sim 10$ ms for ubiquitin.

In the amplitude-modulated MIRROR experiment (Wittmann et al. 2014), rf irradiation is applied on the ${ }^{15} \mathrm{~N}$-channel during the mixing time while the polarization transfer on the protons happens in the laboratory frame leading to similar relaxation times as in the standard laboratory-frame proton spindiffusion experiment. The amplitude-modulation scheme used here was optimized to provide uniform rates over a chemical-shift bandwidth of $\pm 2 \mathrm{kHz}$, which is the relevant range for proton amide-amide correlations at an $850 \mathrm{MHz}$ magnet. For uniform transfer, a maximum rf field of only $2.6 \mathrm{kHz}$ is required on the ${ }^{15} \mathrm{~N}$-channel. Selecting the optimum modulation frequency of the sequence is very important, since it determines the separation of the AM-MIRROR sidebands in the zero-quantum spectrum (Wittmann et al. 2014). As already mentioned above, under our experimental conditions of fast MAS, the full width at half maximum of the zero-quantum line and, thus, also the broadening of the AM-MIRROR sidebands, is small. If a quasi-continuous range of chemical shifts should be recoupled by the sequence, the spacing of the rf sidebands needs to be comparable to their full width at half intensity. Therefore, $v_{\mathrm{m}}$ was set to $200 \mathrm{~Hz}$. As shown in Figure 5C and F, the transfer under AM-MIRROR is considerably more broadband and more efficient than the transfer based on either proton laboratory-frame or proton rotating-frame spin diffusion. Especially for large chemical-shift differences, AM-MIRROR outperforms the other two mixing schemes clearly, such that higher and more uniform polarization-transfer efficiencies are observed. Also in comparison to the first-order recoupling experiments RFDR and DREAM (Figure S4 of the Supporting Information) a less strong alternation of the cross-peak intensities is observed. The performances of the 
two second-order recoupling experiments were further analyzed by comparing the number and intensities of cross-peaks between the rotating-frame spin diffusion and the reverse MIRROR experiment. The peaks from the 3D NNH experiment were assigned on the basis of the published resonance assignment (Agarwal et al. 2014) and are summarized in Figure 6. Besides the expected short-range contacts, several medium $(2<|\Delta N|<4)$ and long-range contacts $(|\Delta N| \geq 5)$ were obtained in both experiments.

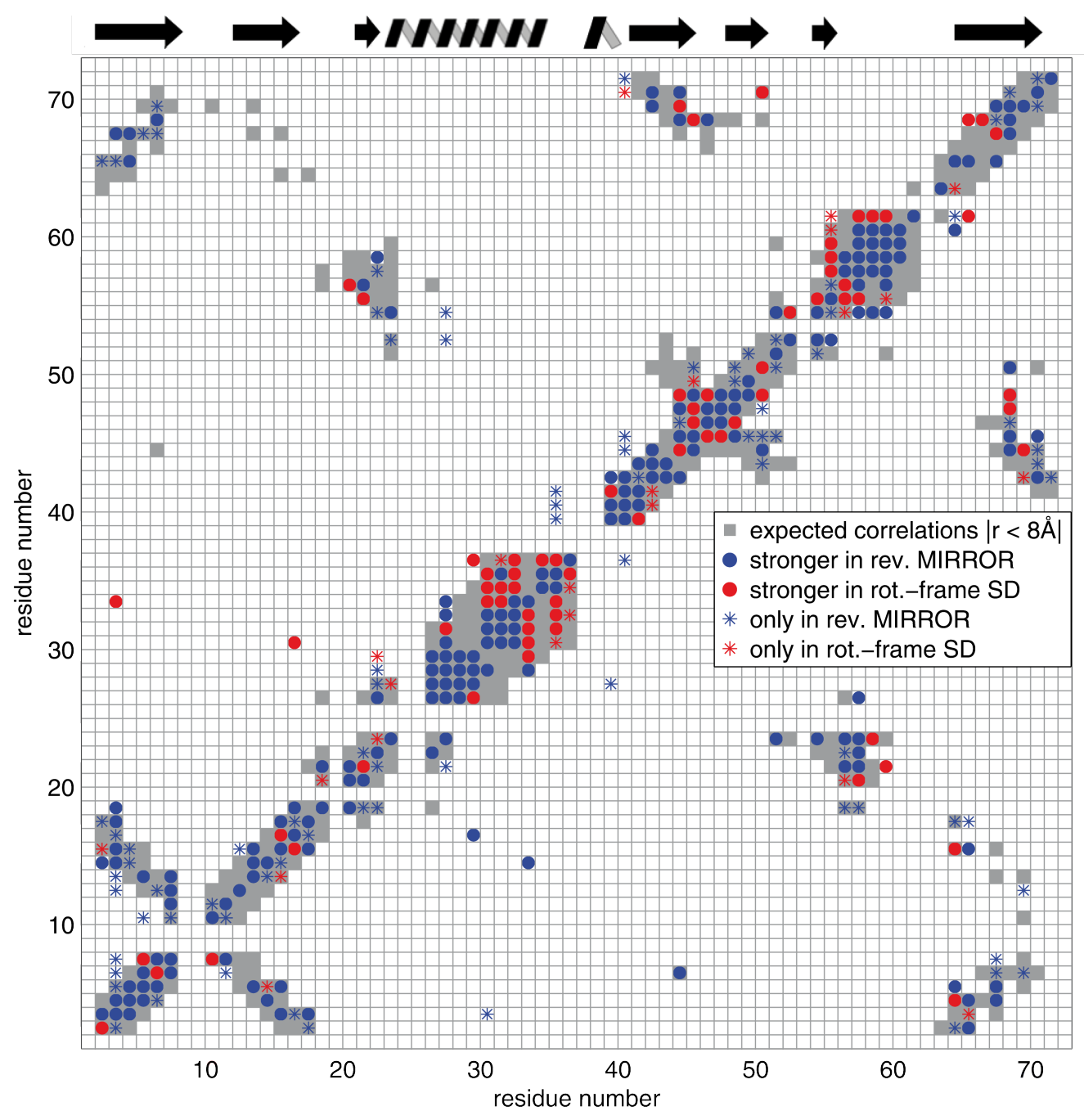

Figure 6: Matrix representation of the experimentally observed $\mathrm{H}^{\mathrm{N}}-\mathrm{H}^{\mathrm{N}}$ contacts under reverse MIRROR (blue) and rotating-frame spin diffusion (red). Cross peaks appearing in both experiments are indicated by a filled circle. Its color is determined by the experiment, where the more intense cross peak is observed (284 in reverse AM-MIRROR, 93 in rotating-frame spin diffusion). Contacts which are found exclusively in one experiment are indicated by asterisks in the respective color. The filled grey squares indicate expected $\mathrm{H}^{\mathrm{N}}-\mathrm{H}^{\mathrm{N}}$ contacts based on the crystal structure (Huang et al. 2011), i.e., internuclear distances less than $8 \AA$. The spectra contain some correlations, which correspond to slightly longer distances than the cut off limit of $8 \AA$. Furthermore, a few contacts corresponding to a transfer over $10 \AA$ 
are observed, which we attribute to relayed transfer. For further information and a full list of the identified correlations, see Table S1 as well as Figure S3 of the Supporting Information.

These cross peaks show a good correlation with the expected $\mathrm{H}^{\mathrm{N}}-\mathrm{H}^{\mathrm{N}}$ contacts from the crystal structure (Huang et al. 2011). For example, several cross peaks can be identified between residues 2 and 27. These contacts depict the anti-parallel orientation of the first two N-terminal $\beta$-sheets. Also correlations between the C-terminal $\beta$-sheet of the protein and residues between Arg42 and Lys48 can be found in the spectra. In most of the cases the transferred polarization is higher in the reverse AM-MIRROR experiment (filled blue circles). Only in the $\alpha$-helical domains, spin diffusion in the rotating frame seems to yield more contacts and more efficient transfer (red circles). However, there are several contacts which can only be found in the reverse AM-MIRROR experiment (blue asterisks). Most prominent are the additional longrange correlations to the first $\mathrm{N}$-terminal $\beta$-sheet.

A comparison of the transfer efficiency of the reverse AM-MIRROR experiment with commonly used first-order recoupling experiments (RFDR and DREAM see Figures S4 to S6 of the Supporting Information) shows that the reverse AM-MIRROR experiment is roughly as efficient as the first-order sequences even at spinning frequencies around $100 \mathrm{kHz}$ with significantly lower rf-field requirements. This is due to the fact that the rf-field amplitude of the AM-MIRROR experiment $(2.6 \mathrm{kHz}$ peak rf-field amplitude) is independent of the MAS frequency while for the first-order recoupling sequences the rffield amplitude increases roughly linearly with the MAS frequency (DREAM requires $v_{1}=v_{\mathrm{r}} / 2$ and RFDR $v_{1} \geq v_{\mathrm{r}}$ ). In addition, the sample heating through $\mathrm{rf}$ is strongly radio-frequency dependent and, therefore, a lot lower at the ${ }^{15} \mathrm{~N}$ Larmor frequencies used in reverse AM-MIRROR. We expect that a second-order recoupling sequence like AM-MIRROR is less susceptible to dipolar truncation effects than first-order recoupling sequences which becomes more important at higher proton densities. The recoupling bandwidth of the AM-MIRROR experiment can be optimized for a given static magnetic field and a defined chemical-shift range leading to the maximum possible and uniform transfer across the desired range. In contrast to this, first-order recoupling sequences are typically broadband across the full range of 
proton chemical shifts. A detailed comparison of the transfer efficiencies depends on the details of the spin system and is beyond the scope of this paper.

\section{Conclusion}

In this publication we demonstrated that for perdeuterated proteins with $100 \%$ protonation at exchangeable sites, proton spin diffusion at MAS conditions of $v_{\text {rot }}>60 \mathrm{kHz}$ is significantly affected by the chemical-shift differences between the proton resonances. Similar to PDSD on ${ }^{13} \mathrm{C}$ spins at fast spinning, the observed transfer is limited to a small range of chemical-shift differences due to the narrowing of the zero-quantum line. In order to compensate these effects, a spin lock can be applied to perform the polarization transfer in the rotating frame. However, the short $T_{1 \rho}$ of protons allows only short mixing times and severely impedes sensitivity. A similar set of distance restraints can be obtained using reverse AM-MIRROR irradiation on the ${ }^{15} \mathrm{~N}$-channel, which is tailored to compensate for offsets in the relevant range of chemical-shift differences. Major advantage of reverse AM-MIRROR compared to the other two approaches is the better efficiency of this recoupling scheme. Furthermore, reverse AMMIRROR is a low-power experiment as the required rf fields do not depend on the MAS frequency but only on the recoupled chemical-shift range. This is in contrast to first-order recoupling sequences like RFDR and DREAM where the rf-field requirement is much higher and increases roughly linearly with the spinning frequency. Together with the fact, that relaxation losses are determined by $T_{1}$, reverse AMMIRROR is a promising candidate for determining proton-proton long-range distance restraints at fast MAS. As demonstrated earlier (Agarwal et al. 2014), the set of amide-amide contacts determined in these experiments only yields a high-resolution protein structure together with additional restraints. Nevertheless, extensions of the proposed recoupling experiment for efficient transfer in samples with different labeling schemes or for fully-protonated proteins have to be developed.

\section{Acknowledgement}


We want to thank Anders Nielsen for the helpful discussions. Nils-Alexander Lakomek and Susanne

Penzel are acknowledged for assistance with the fast-spinning probe. This work has been supported by the Swiss National Science Foundation (grants 200020_146757 and 200020_159797).

\section{References}

Agarwal V, Penzel S, Szekely K, et al (2014) De Novo 3D Structure Determination from Sub-milligram Protein Samples by Solid-State $100 \mathrm{kHz}$ MAS NMR Spectroscopy. Angew Chemie Int Ed 53:12253-12256. doi: 10.1002/anie.201405730

Agarwal V, Tuherm T, Reinhold A, et al (2013) Amplitude-modulated low-power decoupling sequences for fast magic-angle spinning NMR. Chem Phys Lett 583:1-7. doi: 10.1016/j.cplett.2013.07.073

Akbey Ü, Lange S, Trent Franks W, et al (2010) Optimum levels of exchangeable protons in perdeuterated proteins for proton detection in MAS solid-state NMR spectroscopy. J Biomol NMR 46:67-73. doi: 10.1007/s10858-009-9369-0

Andrew ER, Bradbury A, Eades RG (1958) Nuclear Magnetic Resonance Spectra from a Crystal rotated at High Speed. Nature 182:1659-1659.

Bayro MJ, Huber M, Ramachandran R, et al (2009) Dipolar truncation in magic-angle spinning NMR recoupling experiments. J Chem Phys 130:114506. doi: 10.1063/1.3089370

Bennett A, Rienstra C, Griffiths J, et al (1998) Homonuclear radio frequency-driven recoupling in rotating solids. J Chem Phys 108:9463.

Bertini I, Emsley L, Felli IC, et al (2011) High-resolution and sensitivity through-bond correlations in ultra-fast magic angle spinning (MAS) solid-state NMR. Chem Sci 2:345-348. doi: $10.1039 / \mathrm{c} 0 \mathrm{sc} 00397 \mathrm{~b}$

Böckmann A, Ernst M, Meier BH (2015) Spinning proteins, the faster, the better? J Magn Reson 253:7179. doi: 10.1016/j.jmr.2015.01.012

Böckmann A, Gardiennet C, Verel R, et al (2009) Characterization of different water pools in solid-state NMR protein samples. J Biomol NMR 45:319-327. doi: 10.1007/s10858-009-9374-3

Chevelkov V, Rehbein K, Diehl A, Reif B (2006) Ultrahigh Resolution in Proton Solid-State NMR Spectroscopy at High Levels of Deuteration. Angew Chemie Int Ed 45:3878-3881. doi: 10.1002/anie.200600328

De Paëpe G, Lewandowski JR, Loquet A, et al (2008) Proton assisted recoupling and protein structure determination. J Chem Phys 129:245101. doi: 10.1063/1.3036928

Demers J-P, Chevelkov V, Lange A (2011) Progress in correlation spectroscopy at ultra-fast magic-angle spinning: Basic building blocks and complex experiments for the study of protein structure and dynamics. Solid State Nucl Magn Reson 40:101-113. doi: 10.1016/j.ssnmr.2011.07.002 
Deschamps M (2014) Ultrafast Magic Angle Spinning Nuclear Magnetic Resonance. Annu Reports NMR Spectrosc 81:109-144. doi: 10.1016/B978-0-12-800185-1.00003-6

Duma L, Abergel D, Ferrage F, et al (2008) Broadband dipolar recoupling for magnetization transfer in solid-state NMR correlation spectroscopy. ChemPhysChem 9:1104-1106. doi: $10.1002 /$ cphc. 200800053

Ernst M, Meier MA, Tuherm T, et al (2004) Low-Power High-Resolution Solid-State NMR of Peptides and Proteins. J Am Chem Soc 126:4764-4765. doi: 10.1021/ja0494510

Ernst M, Samoson A, Meier BH (2005) Decoupling and recoupling using continuous-wave irradiation in magic-angle-spinning solid-state NMR: a unified description using bimodal Floquet theory. J Chem Phys 123:64102. doi: 10.1063/1.1944291

Grommek A, Meier BH, Ernst M (2006) Distance information from proton-driven spin diffusion under MAS. Chem Phys Lett 427:404-409. doi: 10.1016/j.cplett.2006.07.005

Hohwy M, Rienstra CM, Griffin RG (2002) Band-selective homonuclear dipolar recoupling in rotating solids. J Chem Phys 117:4973. doi: 10.1063/1.1488136

Hou G, Yan S, Sun S, et al (2011) Spin diffusion driven by R-symmetry sequences: applications to homonuclear correlation spectroscopy in MAS NMR of biological and organic solids. J Am Chem Soc 133:3943-53. doi: 10.1021/ja108650x

Hou G, Yan S, Trébosc J, et al (2013) Broadband homonuclear correlation spectroscopy driven by combined R2(n)(v) sequences under fast magic angle spinning for NMR structural analysis of organic and biological solids. J Magn Reson 232:18-30. doi: 10.1016/j.jmr.2013.04.009

Hu B, Lafon O, Trébosc J, et al (2011) Broad-band homo-nuclear correlations assisted by 1H irradiation for bio-molecules in very high magnetic field at fast and ultra-fast MAS frequencies. J Magn Reson 212:320-9. doi: 10.1016/j.jmr.2011.07.011

Huang KY, Amodeo G a., Tong L, McDermott A (2011) The structure of human ubiquitin in 2-methyl2,4-pentanediol: A new conformational switch. Protein Sci 20:630-639. doi: 10.1002/pro.584

Huber M, Hiller S, Schanda P, et al (2011) A proton-detected 4D solid-state NMR experiment for protein structure determination. ChemPhysChem 12:915-918. doi: 10.1002/cphc.201100062

Igumenova TI, McDermott AE, Zilm KW, et al (2004) Assignments of carbon NMR resonances for microcrystalline ubiquitin. J Am Chem Soc 126:6720-6727. doi: 10.1021/ja030547o

Ishii Y (2001) [sup 13]C-[sup 13]C dipolar recoupling under very fast magic angle spinning in solid-state nuclear magnetic resonance: Applications to distance measurements, spectral assignments, and highthroughput secondary-structure determination. J Chem Phys 114:8473. doi: 10.1063/1.1359445

Kubo A, McDowell CA (1988) Spectral spin diffusion in polycrystalline solids under magic-angle spinning. J Chem Soc Faraday Trans 1 84:3713. doi: 10.1039/f19888403713

Kümmerlen J, van Beek JD, Vollrath F, Meier BH (1996) Local Structure in Spider Dragline Silk Investigated by Two-Dimensional Spin-Diffusion Nuclear Magnetic Resonance. Macromolecules 29:2920-2928. doi: 10.1021/ma951098i 
Ladizhansky V (2009) Homonuclear dipolar recoupling techniques for structure determination in uniformly 13C-labeled proteins. Solid State Nucl Magn Reson 36:119-28. doi:

10.1016/j.ssnmr.2009.07.003

Lange A, Luca S, Baldus M (2002) Structural Constraints from Proton-Mediated Rare-Spin Correlation Spectroscopy in Rotating Solids $\uparrow$. J Am Chem Soc 124:9704-9705. doi: 10.1021/ja026691b

Lange A, Scholz I, Manolikas T, et al (2009) Low-power cross polarization in fast magic-angle spinning NMR experiments. Chem Phys Lett 468:100-105. doi: 10.1016/j.cplett.2008.11.089

Lewandowski JR, Dumez JN, Akbey Ü, et al (2011) Enhanced resolution and coherence lifetimes in the solid-state NMR spectroscopy of perdeuterated proteins under ultrafast magic-angle spinning. J Phys Chem Lett 2:2205-2211. doi: 10.1021/jz200844n

Linser R, Bardiaux B, Higman V, et al (2011) Structure calculation from unambiguous long-range amide and methyl $1 \mathrm{H}-1 \mathrm{H}$ distance restraints for a microcrystalline protein with MAS solid-state NMR spectroscopy. J Am Chem Soc 133:5905-12. doi: 10.1021/ja110222h

Lowe IJ (1959) Free induction decays of rotating solids. Phys Rev Lett 2:285-287. doi: 10.1103/PhysRevLett.2.285

Maricq MM, Waugh JS (1979) NMR in rotating solids. J Chem Phys 70:3300. doi: 10.1063/1.437915

Meier BH (1994) Polarization Transfer and Spin Diffusion in Solid-State NMR. Adv Magn Opt Reson 18:1.

Nishiyama Y, Lu X, Trébosc J, et al (2012) Practical choice of 1H-1H decoupling schemes in throughbond $1 \mathrm{H}-\{\mathrm{X}\}$ HMQC experiments at ultra-fast MAS. J Magn Reson 214:151-158. doi: 10.1016/j.jmr.2011.10.014

Parthasarathy S, Nishiyama Y, Ishii Y (2013) Sensitivity and Resolution Enhanced Solid-State NMR for Paramagnetic Systems and Biomolecules under Very Fast Magic Angle Spinning. Acc Chem Res 46:2127-2135. doi: 10.1021/ar4000482

Penzel S, Smith AA, Agarwal V, et al (2015) Protein resonance assignment at MAS frequencies approaching $100 \mathrm{kHz}$ : A quantitative comparison of J-coupling and dipolar-coupling-based transfer methods. J Biomol NMR 63:165-186. doi: 10.1007/s10858-015-9975-y

Reif B (2012) Ultra-high resolution in MAS solid-state NMR of perdeuterated proteins: Implications for structure and dynamics. J Magn Reson 216:1-12. doi: 10.1016/j.jmr.2011.12.017

Robyr P, Meier BH, Ernst RR (1991) Tensor correlation by 2D spin-diffusion powder NMR spectroscopy: determination of the asymmetry of the hydrogen bond potential in benzoic acid. Chem Phys Lett 187:471. doi: 10.1016/0009-2614(91)80285-6

Robyr P, Meier BH, Ernst RR (1989) Radio-frequency-driven nuclear spin diffusion in solids. Chem Phys Lett 162:417-423. doi: 10.1016/0009-2614(89)87001-0

Scholz I, Huber M, Manolikas T, et al (2008) MIRROR recoupling and its application to spin diffusion under fast magic-angle spinning. Chem Phys Lett 460:278-283. doi: 10.1016/j.cplett.2008.05.058 
Scholz I, van Beek JD, Ernst M (2010) Operator-based Floquet theory in solid-state NMR. Solid State Nucl Magn Reson 37:39-59. doi: 10.1016/j.ssnmr.2010.04.003

Senker J, Seyfarth L, Voll J (2004) Determination of rotational symmetry elements in NMR crystallography. Solid State Sci 6:1039-1052. doi: 10.1016/j.solidstatesciences.2004.07.001

Szeverenyi NM, Sullivan MJ, Maciel GE (1982) Observation of spin exchange by two-dimensional fourier transform 13C cross polarization-magic-angle spinning. J Magn Reson 47:462-475. doi: 10.1016/0022-2364(82)90213-X

Takegoshi K, Nakamura S, Terao T (2001) C-13-H-1 dipolar-assisted rotational resonance in magic-angle spinning NMR. Chem Phys Lett 344:631-637.

Takegoshi K, Nakamura S, Terao T (2003) 13C-1H dipolar-driven 13C-13C recoupling without 13C rf irradiation in nuclear magnetic resonance of rotating solids. J Chem Phys 118:2325. doi: $10.1063 / 1.1534105$

Teymoori G, Pahari B, Stevensson B, Edén M (2012) Low-power broadband homonuclear dipolar recoupling without decoupling: Double-quantum 13C NMR correlations at very fast magic-angle spinning. Chem Phys Lett 547:103-109. doi: 10.1016/j.cplett.2012.07.053

Verel R, Ernst M, Meier BH (2001) Adiabatic Dipolar Recoupling in Solid-State NMR: The DREAM Scheme. J Magn Reson 150:81-99. doi: 10.1006/jmre.2001.2310

Veshtort M, Griffin RG (2011) Proton-driven spin diffusion in rotating solids via reversible and irreversible quantum dynamics. J Chem Phys 135:134509. doi: 10.1063/1.3635374

Weingarth M, Bodenhausen G, Tekely P (2009) Broadband carbon-13 correlation spectra of microcrystalline proteins in very high magnetic fields. J Am Chem Soc 131:13937-9. doi: $10.1021 / \mathrm{ja9036143}$

Weingarth M, Bodenhausen G, Tekely P (2010) Broadband magnetization transfer using moderate radiofrequency fields for NMR with very high static fields and spinning speeds. Chem Phys Lett 488:1016. doi: 10.1016/j.cplett.2010.01.072

Wittmann JJ, Hendriks L, Meier BH, Ernst M (2014) Controlling spin diffusion by tailored rf-irradiation schemes. Chem Phys Lett 608:60-67. doi: 10.1016/j.cplett.2014.05.057

Zhou DH, Shah G, Cormos M, et al (2007) Proton-detected solid-state NMR spectroscopy of fully protonated proteins at $40 \mathrm{kHz}$ magic-angle spinning. J Am Chem Soc 129:11791-11801. doi: $10.1021 / \mathrm{ja} 073462 \mathrm{~m}$ 